A new paper published in the American Journal of Transplantation describes the researchers' 5-year experience using this approach in 492 adult renal transplant patients; 120 (24\%) of these patients had a PRA $>30 \%, 58$ of whom had a PRA $>80 \%$. Five-year graft survival was $70 \%, 69 \%$ and $66 \%$ among unsensitized, moderately sensitized (PRA <30\%) and highly sensitized (PRA $>30 \%$ ) patients, respectively, figures comparable with the US national average graft survival rate of $65.7 \%$.

The authors state that this method-the 'Emory Algorithm'-provides a logical and systematic approach for matching recipient and deceased donor kidneys that would improve access for sensitized patients and allow a more equal allocation to this highly disadvantaged group.

Original article Bray RA et al. (2006) Transplanting the highly sensitized patient: the Emory Algorithm. Am J Transplant 6: 2307-2315

\section{LO-Tact-1 reduces chronic rejection and infection following renal transplantation}

A prospective study carried out in France indicates that induction therapy with LO-Tact-1 (rat monoclonal anti-interleukin 2 receptor antibody) might reduce the rate of chronic allograft rejection and incidence of infections following kidney transplantation.

First-time recipients of renal grafts from deceased donors were treated with a quadruple sequential immunosuppressive regimen and randomized to either LO-Tact-1 (10 mg/day; $n=20$ ) or antithymocyte globulin (ATG; $15 \mathrm{mg} /$ day; $n=20$ ) during the first 14 days following transplantation. At the end of the 10-year follow-up period five patients in the LO-Tact-1 group and one patient in the ATG group had died; three deaths occurred within 3 years of transplantation. Mortality rates were $5 \%$ at 2 years and $15 \%$ at 10 years, with no significant difference between the two groups. Death-censored graft loss was lower in patients who received LO-Tact-1 than in those who received ATG, but the difference was not significant. The number of episodes of acute rejection was similar between the two groups. Chronic graft rejection, however, was significantly more frequent in patients treated with ATG than in those treated with LO-Tact-1 (9 vs $3 ; P<0.05$ ) and there were more viral and bacterial infections in the ATG group (8 vs 2 and 16 vs 10, respectively), indicating a benefit of LO-Tact-1 treatment.

Original article Beaudreuil S et al. (2006) Long-term results (10 years) of a prospective trial comparing Lo-tact- 1 monoclonal antibody and anti-thymocyte globulin induction therapy in kidney transplantation. Transpl Int 19: 814-820

\section{Sphygmomanometer calibration could increase the accuracy of hypertension detection}

Poorly calibrated sphygmomanometers could lead to clinically significant errors in hypertension detection, over and above errors introduced as a result of random intra-individual blood pressure (BP) variability. To determine the proportion of error attributable to inadequate sphygmomanometer calibration, Turner et al. carried out a computer simulation study.

The investigators modeled BP measurements in a population of 15 million adult Australians using 17,000 calibrated and 17,000 uncalibrated sphygmomanometers. The simulation showed that, as the number of visits to the clinician increased, the proportion of hypertension-detection error attributable to uncalibrated sphygmomanometers also increased; the effect of this source of error was partially obscured by random BP variability when the number of consultations in an individual was small. After three patient visits, poorly calibrated sphygmomanometers accounted for $20 \%$ of undetected systolic and $28 \%$ of undetected diastolic hypertension (BP >140/90 $\mathrm{mmHg}$ ) and for $15 \%$ of falsely detected systolic and $31 \%$ of falsely detected diastolic hypertension. The model indicated that error attributable to poor sphygmomanometer calibration is more likely to compromise detection of hypertension in young adults (18-24 years old) and women.

More frequent and rigorous calibration of sphygmomanometers by accredited organizations would be a cost-effective way of improving the accuracy of hypertension detection.

Original article Turner MJ et al. (2006) Lack of sphygmomanometer calibration causes over- and underdetection of hypertension: a computer simulation study. J Hypertens 24: 1931-1938 